\title{
BASINS OF ATTRACTION IN LOEWNER EQUATIONS
}

\author{
Leandro Arosio ${ }^{\dagger}$ \\ Istituto Nazionale di Alta Matematica "Francesco Severi" \\ Città Universitaria, Piazzale Aldo Moro 5, 00185 Rome, Italy; arosio@altamatematica.it
}

\begin{abstract}
Let $q \geq 2$. We prove that any Loewner $\mathrm{PDE}$ on the unit ball $\mathbf{B}^{q}$ whose driving term $h(z, t)$ vanishes at the origin and satisfies the bunching condition $\ell m(D h(0, t)) \geq k(D h(0, t))$ for some $\ell \in \mathbf{R}^{+}$, admits a solution given by univalent mappings $\left(f_{t}: \mathbf{B}^{q} \rightarrow \mathbf{C}^{q}\right)_{t \geq 0}$. This is done by discretizing time and considering the abstract basin of attraction. If $\ell<2$, then the range $\cup_{t \geq 0} f_{t}\left(\mathbf{B}^{q}\right)$ of any such solution is biholomorphic to $\mathbf{C}^{q}$.
\end{abstract}

\section{Introduction}

Let $\mathbf{B}^{q} \subset \mathbf{C}^{q}$ denote the unit ball. The Loewner PDE

$$
\frac{\partial f_{t}(z)}{\partial t}=D f_{t}(z) h(z, t) \quad \text { a.e. } t \geq 0, z \in \mathbf{B}^{q}
$$

was introduced by Loewner [21] and developed by Kufarev [20] and Pommerenke [26] in the case of the unit disc $\mathbf{D} \doteq \mathbf{B}^{1}$. The study of this equation culminated with the proof of the Bieberbach conjecture by de Branges [9] and the introduction of the stochastic Loewner evolution by Schramm [27].

The several variables case has been widely studied for its application in geometric function theory by Graham, Hamada, G. Kohr, M. Kohr, Pfaltzgraff and others (see e.g. $[16,25])$.

Throughout this paper we will assume that $q \geq 2$. In [5], generalizing the results obtained in the unit disc $\mathbf{D}$ in [8], we explore the connections between this topic and the theory recently developed by Bracci, Contreras and Díaz-Madrigal [6, 7] (see also [4]) of Herglotz non-autonomous vector fields on complete hyperbolic manifolds. An Herglotz vector field of order $\infty$ on $\mathbf{B}^{q}$ is a non-autonomous holomorphic vector field $-h(z, t): \mathbf{B}^{q} \times \mathbf{R}^{+} \rightarrow \mathbf{C}^{q}$ such that

- $-h(z, t)$ is measurable in $t \geq 0$ and for a.e. $\tilde{t} \geq 0$, the holomorphic vector field $-h(z, \tilde{t})$ is an infinitesimal generator, that is the "frozen" Cauchy problem

$$
\left\{\begin{array}{l}
\dot{z}(s)=-h(z(s), \tilde{t}), \\
z(0)=z_{0},
\end{array}\right.
$$

has a solution $z:[0,+\infty) \rightarrow \mathbf{B}^{q}$ for all $z_{0} \in \mathbf{B}^{q}$,

- for any compact set $K \subset \mathbf{B}^{q}$ and any $T>0$ there exists $c_{K, T}>0$ satisfying

$$
|h(z, t)| \leq c_{K, T}, \quad z \in K, 0 \leq t \leq T .
$$

doi:10.5186/aasfm.2012.3742

2010 Mathematics Subject Classification: Primary 32H50; Secondary 32H02, 37F99.

Key words: Loewner chains in several variables, Loewner equations, evolution families, abstract basins of attraction.

†Titolare di una Borsa della Fondazione Roma - Terzo Settore bandita dall'Istituto Nazionale di Alta Matematica. 
The solution flow of the Loewner ODE

$$
\left\{\begin{array}{l}
\frac{\partial}{\partial t} \varphi_{s, t}(z)=-h\left(\varphi_{s, t}(z), t\right), \quad z \in \mathbf{B}^{q}, \text { a.e. } t \in[s, \infty), \\
\varphi_{s, s}(z)=z, \quad z \in \mathbf{B}^{q}, s \geq 0
\end{array}\right.
$$

is an evolution family of order $\infty$, that is a family of holomorphic mappings $\left(\varphi_{s, t}\right.$ : $\mathbf{B}^{q}$ $\left.\rightarrow \mathbf{B}^{q}\right)_{0 \leq s \leq t}$ satisfying

- $\varphi_{s, t}=\varphi_{u, t} \circ \varphi_{s, u}$ for all $0 \leq s \leq u \leq t$ and $\varphi_{s, s}(z)=z$ for all $s \geq 0$,

- for any compact set $K \subset \mathbf{B}^{q}$ and for any $T>0$ there exists a $C_{K, T}>0$ satisfying

$$
\left|\varphi_{s, t}(z)-\varphi_{s, u}(z)\right| \leq C_{K, T}(t-u), \quad z \in K, 0 \leq s \leq u \leq t<T .
$$

In [5] we prove that a family $\left(f_{t}: \mathbf{B}^{q} \rightarrow \mathbf{C}^{q}\right)_{t \geq 0}$ of univalent mappings is locally Lipschitz (in the variable $t$ ) and solves the Loewner PDE (1.1) if and only if it solves the functional equation

$$
f_{s}=f_{t} \circ \varphi_{s, t}, \quad 0 \leq s \leq t .
$$

If such a solution $\left(f_{t}: \mathbf{B}^{q} \rightarrow \mathbf{C}^{q}\right)$ exists, then the subset $\bigcup_{t \geq 0} f_{t}\left(\mathbf{B}^{q}\right) \subset \mathbf{C}^{q}$ is open and connected and is called the range of $\left(f_{t}\right)$. Any other solution $\left(g_{t}: \mathbf{B}^{q} \rightarrow \mathbf{C}^{q}\right)$ is of the form $\left(\Lambda \circ f_{t}\right)$, where $\Lambda: \bigcup_{t>0} f_{t}\left(\mathbf{B}^{q}\right) \rightarrow \mathbf{C}^{q}$ is holomorphic. Thus the ranges of two univalent solutions of (1.1) are biholomorphic.

We are interested in Herglotz vector fields on $\mathbf{B}^{q}$ whose flow $\left(\varphi_{s, t}\right)$ is attracting at the origin. A first example is provided by Herglotz vector fields whose linear part does not depend on $t \geq 0$. This has been studied in $[10,14]$.

Theorem 1.1. Let $-h(z, t)$ be a Herglotz vector field of order $\infty$ on $\mathbf{B}^{q}$ such that $h(z, t)=A z+O\left(|z|^{2}\right)$ with

$$
2 \min \{\operatorname{Re}\langle A z, z\rangle:|z|=1\}>\max \{\operatorname{Re} \lambda: \lambda \in \operatorname{sp}(A)\}
$$

where $\langle\cdot, \cdot\rangle$ is the hermitian product on $\mathbf{C}^{q}$. Then the Loewner PDE (1.1) admits a locally Lipschitz univalent solution $\left(f_{t}: \mathbf{B}^{q} \rightarrow \mathbf{C}^{q}\right)$. The range $\bigcup_{t \geq 0} f_{t}\left(\mathbf{B}^{q}\right)$ of any such solution is biholomorphic to $\mathbf{C}^{q}$.

This result was generalized in [3] (see also [2]), with an approach based on a discretization of time.

Theorem 1.2. Let $-h(z, t)$ be a Herglotz vector field of order $\infty$ on $\mathbf{B}^{q}$ such that $h(z, t)=A z+O\left(|z|^{2}\right)$, where the eigenvalues of $A$ have strictly positive real part. Then the Loewner PDE (1.1) admits a locally Lipschitz univalent solution $\left(f_{t}: \mathbf{B}^{q} \rightarrow \mathbf{C}^{q}\right)$. The range $\bigcup_{t \geq 0} f_{t}\left(\mathbf{B}^{q}\right)$ of any such solution is biholomorphic to $\mathbf{C}^{q}$.

The same result was obtained independently with different methods by Voda [28], assuming $\min \{\operatorname{Re}\langle A z, z\rangle:|z|=1\}>0$. See also [18] for related results.

The next natural step is admitting time-dependent linear parts. Set

$$
m(A) \doteq \min \{\operatorname{Re}\langle A z, z\rangle:|z|=1\}, \quad k(A)=\max \{\operatorname{Re}\langle A z, z\rangle:|z|=1\} .
$$

The following result is proved in $[15,13]$ :

Theorem 1.3. Let $-h(z, t)$ be a Herglotz vector field on $\mathbf{B}^{q}$ of order $\infty$ such that $h(z, t)=A(t) z+O\left(|z|^{2}\right)$, and assume that the family of linear mappings $(A(t))_{t \geq 0}$ satisfies:

i) $m(A(t))>0$ for all $t \geq 0$ and $\int_{0}^{\infty} m(A(t)) d t=\infty$, 
ii) $t \mapsto\|A(t)\|$ is uniformly bounded on $\mathbf{R}^{+}$,

iii) there exists $\delta>0$ such that

$$
2 m(A(t)) \geq k(A(t))+\delta, \quad t \geq 0,
$$

iv)

$$
\int_{s}^{t} A(\tau) d \tau \circ \int_{r}^{s} A(\tau) d \tau=\int_{r}^{s} A(\tau) d \tau \circ \int_{s}^{t} A(\tau) d \tau, \quad t \geq s \geq r \geq 0 .
$$

Then the Loewner PDE (1.1) admits a locally Lipschitz univalent solution $\left(f_{t}: \mathbf{B}^{q} \rightarrow\right.$ $\left.\mathbf{C}^{q}\right)$. The range $\bigcup_{t \geq 0} f_{t}\left(\mathbf{B}^{q}\right)$ of any such solution is biholomorphic to $\mathbf{C}^{q}$.

In this paper we generalize Theorem 1.3, using the approach of [2][3]. The following is our result.

Theorem 1.4. Let $-h(z, t)$ be a Herglotz vector field on $\mathbf{B}^{q}$ of order $\infty$ such that $h(z, t)=A(t) z+O\left(|z|^{2}\right)$, and assume that the family of linear mappings $(A(t))_{t \geq 0}$ satisfies:

a) $m(A(t))>0$ for all $t \geq 0$ and $\int_{0}^{\infty} m(A(t)) d t=\infty$,

b) $t \mapsto\|A(t)\|$ is locally bounded on $\mathbf{R}^{+}$,

c) there exists $\ell \in \mathbf{R}^{+}$such that

$$
\ell m(A(t)) \geq k(A(t)), \quad t \geq 0 .
$$

Then the Loewner PDE (1.1) admits a locally Lipschitz univalent solution $\left(f_{t}: \mathbf{B}^{q} \rightarrow\right.$ $\left.\mathbf{C}^{q}\right)$. If $\ell<2$ then the range $\bigcup_{t \geq 0} f_{t}\left(\mathbf{B}^{q}\right)$ of any such solution is biholomorphic to $\mathrm{C}^{q}$.

Notice that the assumptions ii) and iii) of Theorem 1.3 imply that there exists $\ell<2$ such that

$$
\ell m(A(t)) \geq k(A(t)), \quad t \geq 0 .
$$

We want to stress the strong analogy between Loewner theory and the theory of discrete non-autonomous complex dynamical systems which has developed around Bedford's conjecture (see $[1,11,19,23,30]$ ). This is reflected in the proof of Theorem 1.4, which is based on a discretization of time, and relies on the study of the abstract basin of attraction performed by Fornaess and Stensønes in [11]:

Theorem 1.5. Let $\left(\varphi_{n, n+1}\right)_{n \in \mathbf{N}}$ be a family of univalent self-mappings of $r \mathbf{B}^{q}$. Assume that there exist $0<\nu \leq \mu<1$ such that

$$
\nu|z| \leq\left|\varphi_{n, n+1}(z)\right| \leq \mu|z|, \quad z \in r \mathbf{B}^{q}, n \in \mathbf{N} .
$$

Then, if $\Omega$ is the abstract basin of attraction of $\left(\varphi_{n, n+1}\right)$, then there exists an univalent mapping $\Psi: \Omega \rightarrow \mathbf{C}^{q}$.

The abstract basin of attraction comes naturally with a family of univalent mappings $\left(\omega_{n}: r \mathbf{B}^{q} \rightarrow \Omega\right)$. Composing this family with the biholomorphism $\Psi$ given by Theorem 1.5 we obtain a family of univalent mappings from $r \mathbf{B}$ to $\mathbf{C}^{q}$ which we extend to a family $\left(f_{t}: \mathbf{B}^{q} \rightarrow \mathbf{C}^{q}\right)$ satisfying the functional equation (1.4).

The range $\bigcup_{t \geq 0} f_{t}\left(\mathbf{B}^{q}\right)$ is by construction biholomorphic to $\Omega$ and thus by [11, Theorem 3.1] it is a Stein, Runge domain in $\mathbf{C}^{q}$ whose Kobayashi pseudometric vanishes identically and which is diffeomorphic to $\mathbf{C}^{q}$. It is an open question whether $\bigcup_{t \geq 0} f_{t}\left(\mathbf{B}^{q}\right)$ is biholomorphic to $\mathbf{C}^{q}$ when $\ell \geq 2$. A positive answer would follow from a proof of Bedford's conjecture (see e.g. [22]): 
Conjecture 1.6. Let $\left(\Phi_{n, n+1}\right)_{n \in \mathbf{N}}$ be a family of automorphisms of $\mathbf{C}^{q}$. Assume that there exist $0<\nu \leq \mu<1$ and $r>0$ such that

$$
\nu|z| \leq\left|\Phi_{n, n+1}(z)\right| \leq \mu|z|, \quad z \in r \mathbf{B}^{q}, n \in \mathbf{N} .
$$

Then the basin of attraction

$$
\left\{z \in \mathbf{C}^{q}: \lim _{n \rightarrow \infty} \Phi_{n-1, n} \circ \cdots \circ \Phi_{0,1}=0\right\}
$$

is biholomorphic to $\mathbf{C}^{q}$.

I want to thank Jasmin Raissy and Han Peters for our valuable discussions. I also want to thank the referee for precious comments.

\section{Main result}

Let $\mathcal{N}$ denote the family of holomorphic mappings $h: \mathbf{B}^{q} \rightarrow \mathbf{C}^{q}$ such that $h(0)=$ 0 and $\operatorname{Re}\langle h(z), z\rangle>0$, for all $z \neq 0$.

Theorem 2.1. Let $h(z, t): \mathbf{B}^{q} \times \mathbf{R}^{+} \rightarrow \mathbf{C}^{q}$ be a mapping such that $z \mapsto h(z, t) \in$ $\mathcal{N}$ for all $t \in \mathbf{R}^{+}$and $t \mapsto h(z, t)$ is measurable on $\mathbf{R}^{+}$for all $z \in \mathbf{B}^{q}$. Assume that $h(z, t)=A(t) z+O\left(|z|^{2}\right)$ and that the family of linear mappings $(A(t))_{t \geq 0}$ satisfies:

a) $m(A(t))>0$ for all $t \geq 0$ and $\int_{0}^{\infty} m(A(t)) d t=\infty$,

b) $t \mapsto\|A(t)\|$ is locally bounded on $\mathbf{R}^{+}$,

c) there exists $\ell \in \mathbf{R}^{+}$such that

$$
\ell m(A(t)) \geq k(A(t)), \quad t \geq 0 .
$$

Then the Loewner PDE

$$
\frac{\partial f_{t}(z)}{\partial t}=D f_{t}(z) h(z, t), \quad z \in \mathbf{B}^{q}, \text { a.e. } t \geq 0
$$

admits a locally Lipschitz solution given by univalent mappings $\left(f_{t}: \mathbf{B}^{q} \rightarrow \mathbf{C}^{q}\right)$. If $l<2$, then the range $\bigcup_{t>0} f_{t}\left(\mathbf{B}^{q}\right)$ of any such solution is biholomorphic to $\mathbf{C}^{q}$. Any other solution given by holomorphic mappings $\left(g_{t}: \mathbf{B}^{q} \rightarrow \mathbf{C}^{q}\right)$ is of the form $\left(\Lambda \circ f_{t}\right)$, where $\Lambda: \bigcup_{t \geq 0} f_{t}\left(\mathbf{B}^{q}\right) \rightarrow \mathbf{C}^{q}$ is holomorphic.

Proof. Notice that for all $A \in \mathcal{L}\left(\mathbf{C}^{q}\right)$,

$$
m(A) \leq k(A) \leq\|A\|,
$$

and thus $k(t)$ and $m(t)$ are also locally bounded on $\mathbf{R}^{+}$. By [14, Lemma 1.2] one has for a.e. $t \geq 0$

$$
|h(z, t)| \leq \frac{4 r}{(1-r)^{2}}\|A(t)\|, \quad|z| \leq r<1,
$$

hence $-h(z, t)$ is a Herglotz vector field of order $\infty$ on $\mathbf{B}^{q}$. Let $\left(\varphi_{s, t}\right)$ be the associated evolution family of order $\infty$, that is the solution of the Loewner ODE

$$
\left\{\begin{array}{l}
\frac{\partial}{\partial t} \varphi_{s, t}(z)=-h\left(\varphi_{s, t}(z), t\right), \quad z \in \mathbf{B}^{q}, \text { a.e. } t \in[s, \infty), \\
\varphi_{s, s}(z)=z, \quad z \in \mathbf{B}^{q}, s \geq 0 .
\end{array}\right.
$$

Recall that $\varphi_{s, t}: \mathbf{B}^{q} \rightarrow \mathbf{B}^{q}$ is an univalent mapping for all $0 \leq s \leq t$ and that $t \mapsto \varphi_{s, t}(z)$ is locally Lipschitz continuous on $[s, \infty)$ uniformly on compact sets with respect to $z \in \mathbf{B}^{q}$. 
Fix $s \geq 0$ and $z \in \mathbf{B}^{q} \backslash\{0\}$. Then for a.e. $\tau \geq s$,

$$
\frac{\partial}{\partial \tau}\left|\varphi_{s, \tau}(z)\right|^{2}=2 \operatorname{Re}\left\langle\frac{\partial}{\partial \tau} \varphi_{s, \tau}(z), \varphi_{s, \tau}(z)\right\rangle=-2 \operatorname{Re}\left\langle h\left(\varphi_{s, \tau}(z), \tau\right), \varphi_{s, \tau}(z)\right\rangle .
$$

Set $C(r) \doteq \frac{1+r}{1-r}$ and $c(r) \doteq \frac{1-r}{1+r}$ for all $r \geq 0$. Gurganus proved [17] that for a.e. $t \geq 0$,

$\operatorname{Re}\langle A(t) w, w\rangle c(|w|) \leq \operatorname{Re}\langle h(w, t), w\rangle \leq \operatorname{Re}\langle A(t) w, w\rangle C(|w|), \quad w \in \mathbf{B}^{q} \backslash\{0\}$.

Since $\left|\varphi_{s, \tau}(z)\right| \leq|z|$ one has

$$
\begin{gathered}
-2 k(A(\tau)) C(|z|) \leq \frac{\frac{\partial}{\partial \tau}\left|\varphi_{s, \tau}(z)\right|^{2}}{\left|\varphi_{s, \tau}(z)\right|^{2}} \leq-2 m(A(\tau)) c(|z|), \quad \text { a.e. } \tau \geq 0, \\
-2 C(|z|) \int_{s}^{t} k(A(\tau)) d \tau \leq \int_{s}^{t} \frac{\frac{\partial}{\partial \tau}\left|\varphi_{s, \tau}(z)\right|^{2}}{\left|\varphi_{s, \tau}(z)\right|^{2}} d \tau \leq-2 c(|z|) \int_{s}^{t} m(A(\tau)) d \tau, \quad 0 \leq s \leq t, \\
(2.2) \quad e^{-C(|z|) \int_{s}^{t} k(A(\tau)) d \tau} \leq \frac{\left|\varphi_{s, t}(z)\right|}{|z|} \leq e^{-c(|z|) \int_{s}^{t} m(A(\tau)) d \tau}, \quad 0 \leq s \leq t .
\end{gathered}
$$

Set for all $0 \leq s \leq t$,

$$
\nu_{s, t} \doteq e^{-C(|z|) \int_{s}^{t} k(A(\tau)) d \tau}, \quad \text { and } \quad \mu_{s, t} \doteq e^{-c(|z|) \int_{s}^{t} m(A(\tau)) d \tau} .
$$

One has, thanks to assumption c),

$$
\log _{\mu_{s, t}} \nu_{s, t}=\frac{\log \nu_{s, t}}{\log \mu_{s, t}}=C^{2}(|z|) \frac{\int_{s}^{t} k(A(\tau)) d \tau}{\int_{s}^{t} m(A(\tau)) d \tau} \leq C^{2}(|z|) \ell, \quad 0 \leq s \leq t .
$$

Let $n \in \mathbf{N}$ and let $u_{n} \in \mathbf{R}^{+}$be defined by

$$
\int_{0}^{u_{n}} m(A(\tau)) d \tau=n
$$

Let now $h \in \mathbf{N}$ be the least integer strictly greater than $\ell$, and let $r>0$ be such that $C^{2}(r)<h / \ell$. Set $\mu \doteq e^{-c(r)}$ (notice that $\mu=\mu_{u_{n}, u_{n+1}}$ for all $n \geq 0$ ) and $\nu \doteq \min \left\{\nu_{u_{n}, u_{n+1}}: n \geq 0\right\}$. By (2.2) and (2.3) one has that

$$
\nu|z| \leq\left|\varphi_{u_{n}, u_{n+1}}(z)\right| \leq \mu|z|, \quad z \in r \mathbf{B}^{q}, n \geq 0
$$

and

$$
\mu^{h}<\nu
$$

The abstract basin of attraction or tail space $\Omega$ of the family $\left(\varphi_{u_{n}, u_{n+1}}: r \mathbf{B}^{q} \rightarrow\right.$ $r \mathbf{B}^{q}$ ) is defined in [11] (see also [1]) as its topological inductive limit endowed with a natural complex structure. $\Omega$ is the quotient of the set

$$
\left\{z \in \prod_{m \geq n} r \mathbf{B}^{q}: n \in \mathbf{N}, z_{m+1}=\varphi_{u_{m}, u_{m+1}}\left(z_{m}\right), \quad 0 \leq n \leq m\right\},
$$

obtained identifying $z$ and $z^{\prime}$ if $z_{m}=z_{m}^{\prime}$ for $m$ large enough, and the holomorphic structure is induced by a family of open inclusions $\left(\omega_{n}: r \mathbf{B}^{q} \rightarrow \Omega\right)$ defined as

$$
\omega_{n}(z) \doteq\left(\varphi_{u_{n}, u_{m}}(z)\right)_{m \geq n}, \quad n \in \mathbf{N}
$$

which are thus by definition biholomorphisms with their image and satisfy

$$
\omega_{n}(z)=\omega_{m} \circ \varphi_{u_{n}, u_{m}}(z), \quad 0 \leq n \leq m, z \in r \mathbf{B}^{q} .
$$


By [11, Theorem 2.2] there exists an univalent mapping $\Psi: \Omega \rightarrow \mathbf{C}^{q}$. We claim that, for all $s \geq 0$, the sequence $\left(\Psi \circ \omega_{m} \circ \varphi_{s, u_{m}}\right)_{m \geq 0}$ converges uniformly on compact sets in $\operatorname{Hol}\left(\mathbf{B}^{q}, \mathbf{C}^{q}\right)$. Indeed by equation (2.2) and assumption a) one has that for all $s \geq 0$,

$$
\lim _{m \rightarrow \infty} \varphi_{s, u_{m}}(z)=0
$$

uniformly on compact sets. Thus, if $0<v<1$, there exist $m(v) \in \mathbf{N}$ such that for all $j \geq m(v)$, one has $\varphi_{s, u_{j}}\left(v \mathbf{B}^{q}\right) \subset r \mathbf{B}^{q}$. Let $j, h$ be integers such that $m(v) \leq j \leq h$, then by $(2.5)$,

$$
\Psi \circ \omega_{h} \circ \varphi_{s, u_{h}}(z)=\Psi \circ \omega_{j} \circ \varphi_{s, u_{j}}(z), \quad z \in v \mathbf{B}^{q} .
$$

Thus the sequence $\left(\Psi \circ \omega_{m} \circ \varphi_{s, u_{m}}\right)$ is eventually constant in $\operatorname{Hol}\left(v \mathbf{B}^{q}, \mathbf{C}^{q}\right)$.

Let $f_{t}: \mathbf{B}^{q} \rightarrow \mathbf{C}^{q}$ the univalent mapping defined as

$$
f_{t}(z) \doteq \lim _{m \rightarrow+\infty} \Psi \circ \omega_{m} \circ \varphi_{t, u_{m}}(z) .
$$

One easily verifies that

$$
f_{s}(z)=f_{t} \circ \varphi_{s, t}(z), \quad 0 \leq s \leq t, z \in \mathbf{B}^{q},
$$

and that

$$
\bigcup_{t \geq 0} f_{t}\left(\mathbf{B}^{q}\right)=\Psi(\Omega)
$$

Notice that the abstract basin of attraction of the family $\left(\varphi_{u_{n}, u_{n+1}}\right)$ is thus biholomorphic to the Loewner range of the family $\left(\varphi_{s, t}\right)$ defined in [5]. This can be checked directly since both objects are defined as direct limits. that is

By [5, Theorem 4.10] one has that $\left(f_{t}: \mathbf{B}^{q} \rightarrow \mathbf{C}^{q}\right)$ is a Loewner chain of order $\infty$,

- $f_{s}\left(\mathbf{B}^{q}\right) \subset f_{t}\left(\mathbf{B}^{q}\right)$ for all $0 \leq s \leq t$,

- for any compact set $K \subset \mathbf{B}^{q}$ and for any $T>0$ there exists a $k_{K, T}>0$ satisfying

$$
\left|f_{t}(z)-f_{s}(z)\right| \leq k_{K, T}(t-s), \quad z \in K, 0 \leq s \leq t<T .
$$

By [5, Theorem 5.2] one obtains finally

$$
\frac{\partial f_{t}(z)}{\partial t}=D f_{t}(z) h(z, t), \quad z \in \mathbf{B}^{q} \text {, a.e. } t \geq 0 \text {. }
$$

Thus any univalent mapping $\Psi: \Omega \rightarrow \mathbf{C}^{q}$ gives rise to a univalent solution $\left(f_{t}: \mathbf{B}^{q} \rightarrow \mathbf{C}^{q}\right)$ of the Loewner PDE. Following [1, Remark A.4] we recall a way to construct such a univalent mapping $\Psi: \Omega \rightarrow \mathbf{C}^{q}$. Given any polynomial map $p: \mathbf{C}^{q} \rightarrow \mathbf{C}^{q}$ of degree at most $k$ with $D p(0)$ invertible there exists [12][29] an holomorphic automorphism $\Phi$ of $\mathbf{C}^{q}$ such that

$$
\Phi(z)=p(z)+O\left(|z|^{k+1}\right) .
$$

We choose a sequence of automorphisms $\left(\Phi_{n, n+1}: \mathbf{C}^{q} \rightarrow \mathbf{C}^{q}\right)$ which is uniformly bounded on a neighborhood of the origin and which satisfies

$$
\Phi_{n, n+1}(z)=\varphi_{u_{n}, u_{n+1}}(z)+O\left(|z|^{h}\right), \quad n \geq 0,
$$

where $h \in \mathbf{N}$ is as in (2.4). We denote the basin of attraction of the sequence $\left(\Phi_{n, n+1}\right)$ by

$$
\mathfrak{A}\left(\Phi_{n, n+1}\right) \doteq\left\{z \in \mathbf{C}^{q}: \lim _{n \rightarrow \infty} \Phi_{n-1, n} \circ \cdots \circ \Phi_{0,1}=0\right\} .
$$


It follows from [1, Theorem A.1] that there exists a biholomorphism

$$
\Psi: \Omega \rightarrow \mathfrak{A}\left(\Phi_{n, n+1}\right) \subset \mathbf{C}^{q} .
$$

If $\ell<2$, then $h=2$ and, by [30, Theorem 4], one has that the basin of attraction $\mathfrak{A}\left(\Phi_{n, n+1}\right)$ is biholomorphic to $\mathbf{C}^{q}$.

By [5, Theorem 4.10] any solution $\left(g_{t}: \mathbf{B}^{q} \rightarrow \mathbf{C}^{q}\right)$ of the Loewner PDE has to satisfy $g_{s}=g_{t} \circ \varphi_{s, t}$ for all $0 \leq s \leq t$ and thus [5, Theorem 4.7] yields that the family $\left(g_{t}\right)$ is of the form $\left(\Lambda \circ f_{t}\right)$, where $\Lambda: \bigcup_{t \geq 0} f_{t}\left(\mathbf{B}^{q}\right) \rightarrow \mathbf{C}^{q}$ is holomorphic.

\section{References}

[1] Abate, M., A. Abbondandolo, and P. Majer: Stable manifolds for holomorphic automorphisms. - Preprint, arXiv:1104.4561v2 [math.DS].

[2] Arosio, L.: Resonances in Loewner equations. - Adv. Math. 227, 2011, 1413-1435.

[3] Arosio, L.: Loewner equations on complete hyperbolic domains. - Preprint, arXiv:1102.5454 [math.CV].

[4] Arosio, L., and F. BracCI: Infinitesimal generators and the Loewner equation on complete hyperbolic manifolds. - Anal. Math. Phys. 1:4, 2011, 337-350.

[5] Arosio, L., F. Bracci, H. Hamada, and G. Kohr: An abstract approach to Loewner chains. - J. Anal. Math. (to appear), arXiv:1002.4262v1 [math.CV].

[6] Bracci, F., M. D. Contreras, and S. Díaz-Madrigal: Evolution families and the Loewner equation I: the unit disc. - J. Reine Angew. Math. (to appear), DOI:10.1515/crelle.2011.167.

[7] Bracci, F., M. D. Contreras, and S. Díaz-Madrigal: Evolution families and the Loewner equation II: complex hyperbolic manifolds. - Math. Ann. 344, 2009, 947-962

[8] Contreras, M. D., S. Díaz-Madrigal, and P. Gumenyuk: Loewner chains in the unit disc. - Rev. Mat. Iberoamericana 26, 2010, 975-1012.

[9] De Branges, L.: A proof of the Bieberbach conjecture. - Acta Math. 154, 1985, 137-152.

[10] Duren, P., I. Graham, H. Hamada, and G. Kohr: Solutions for the generalized Loewner differential equation in several complex variables. - Math. Ann. 347:2, 2010, 411-435.

[11] Fornaess, J.E., and B. Stensønes: Stable manifolds of holomorphic hyperbolic maps. Internat. J. Math. 15, 2004, 749-758

[12] Forstneric, F.: Interpolation by holomorphic automorphisms and embeddings in $\mathbf{C}^{n}$. - J. Geom. Anal. 9, 1999, 93-117.

[13] Graham, I., H. Hamada, and G. Kohr: On subordination chains with normalization given by a time-dependent linear operator. - Complex Anal. Oper. Theory 5:3, 2011, 787-797.

[14] Graham, I., H. Hamada, G. Kohr, and M. Kohr: Asymptotically spirallike mappings in several complex variables. - J. Anal. Math. 105, 2008, 267-302.

[15] Graham, I., H. Hamada, G. Kohr, and M. Kohr: Spirallike mappings and univalent subordination chains in $\mathbf{C}^{n}$. - Ann. Sc. Norm. Super. Pisa Cl. Sci. (5), 2008, 717-740.

[16] Graham, I., and G. Kohr: Geometric function theory in one and higher dimensions. - Marcel Dekker Inc., New York, 2003.

[17] Gurganus, K.: $\Psi$-like holomorphic functions in $\mathbf{C}^{n}$ and Banach spaces. - Trans. Amer. Math. Soc. 205, 1975, 389-406.

[18] Hamada, H.: Polynomially bounded solutions to the Loewner differential equation in several complex variables. - J. Math. Anal. Appl. 381, 2011, 179-186.

[19] Jonsson, M., and D. VArolin: Stable manifolds of holomorphic diffeomorphisms. - Invent. Math. 149:2, 2002, 409-430. 
[20] Kufarev, P. P.: On one-parameter families of analytic functions. - Mat. Sb. 13, 1943, 87-118 (in Russian).

[21] Loewner, C.: Untersuchungen über schlichte konforme Abbildungen des Einheitskreises. Math. Ann. 89, 1923, 103-121.

[22] Peters, H.: Non-autonomous complex dynamical systems. - Ph.D. thesis, Univ. of Michigan, 2005.

[23] Peters, H.: Perturbed basins of attraction. - Math. Ann. 337, 2007, 1-13.

[24] Peters, H., and E. F. Wold: Non-autonomous basins of attraction and their boundaries. J. Geom. Anal. 15, 2005, 123-136.

[25] Pfaltzgraff, J. A.: Subordination chains and univalence of holomorphic mappings in $\mathbf{C}^{n}$. Math. Ann. 210, 1974, 55-68.

[26] Pommerenke, Ch.: Über die Subordination analytischer Funktionen. - J. Reine Angew. Math. 218, 1965, 159-173.

[27] Schramm, O.: Scaling limits of loop-erased random walks and uniform spanning trees. - Israel J. Math. 118, 2000, 221-288.

[28] VodA, M.: Solution of a Loewner chain equation in several variables. - J. Math. Anal. Appl. $375: 1,2011,58-74$.

[29] Weickert, B.: Automorphisms of $\mathbf{C}^{n}$. - Ph.D. thesis, Univ. of Michigan, 1997.

[30] Wold, E. F.: Fatou-Bieberbach domains. - Internat. J. Math. 16, 2005, 1119-1130.

Received 17 February 2012 